\title{
Efeitos de Crescimento e Bem-estar da Lei de Parceria Público-Privada no Brasil*
}

\author{
Ricardo A. de Castro Pereira ${ }^{\dagger}$, Pedro Cavalcanti Ferreira ${ }^{\ddagger}$
}

Sumário: 1. Introdução; 2. O Modelo; 3. Calibração; 4. Resultados; 5. Conclusão;

Palavras-chave: Infra-Estrutura; Parceria Público-Privada; Crescimento; Modelos Recursivos.

Códigos JEL: E62; H54; E37.

Este artigo utiliza um modelo dinâmico de equilíbrio geral para investigar os impactos de crescimento econômico e bem estar associados à política de parceria público-privada (PPP) no Brasil. Assume-se uma economia com capital privado e infra-estrutura pública e privada e um governo que, além de investir, arrecada impostos, recebe renda de seus serviços e transfere renda para os indivíduos. O modelo é calibrado para a economia brasileira, buscando reproduzir os mecanismos da Lei 11.079, de dezembro de 2004, que criou a PPP no Brasil. Especial atenção foi dada à mensuração da parcela dos serviços públicos de infra-estrutura que não é paga pelos indivíduos e para estimação das alíquotas dos diferentes impostos. As simulações indicam que o impacto potencial da Lei das PPPs sobre o crescimento é pouco significativo. Entretanto, a magnitude dos ganhos de bem-estar pode ser expressiva, dependendo do efeito externo da infra-estrutura, um parâmetro de mensuração delicada.

This article investigates the growth and welfare impacts of the Public-PrivatePartnership (PPP) policy in Brazil, by means of a recursive model calibrated to the country. The theoretical model assumes two types of capital - physical and infrastructure - and the latter may by public or private. Our experiment reproduces the main features of the PPP law, the Law $n^{0} 11.079$ of December 2004. In the calibration process, we paid special attention to the fraction of infrastructure services supplied for free by the government and to the calculation of tariffs and taxes. Simulations found that the potential impact on growth and welfare are not substantial. Nevertheless, those magnitudes could be magnified depending on the external effect of infrastructure.

\footnotetext{
*Os autores gostariam de agradecer os comentários de Samuel Pessoa, Ricardo Cavalcanti, Arilton Teixeira, Sérgio Ferreira e pareceristas. R. A. C. Pereira agradece o financiamento da Capes e P. C. Ferreira o financiamento do CNPq e Faperj.

${ }^{\dagger}$ Curso de Pós-Graduação em Economia, Universidade Federal do Ceará. E-mail: rpereira@caen.ufc.br

‡Escola de Pós-Graduação em Economia, Fundação Getulio Vargas. E-mail: ferreira@fgv.br
} 


\section{INTRODUÇÃO}

O objetivo deste artigo é investigar quantitativamente o potencial das Parcerias Público-Privadas (PPPs) no Brasil em promover o crescimento econômico e bem estar social, admitindo-se um ambiente restrito pelas regras impostas por sua Lei (Lei número 11.079 de 30 de dezembro de 2004). Especificamente, avalia-se o mecanismo das PPPs como estímulo aos investimentos privados em infra-estrutura, utilizando-se um modelo de equilíbrio geral competitivo derivado do modelo neoclássico de crescimento.

De maneira simplificada, PPPs são contratos envolvendo pagamentos sistemáticos do setor público ao parceiro privado em contrapartida à oferta de serviços que, originalmente são ofertados pelo primeiro, devido, por exemplo, aos seus retornos sociais serem superiores aos retornos privados. 0 compromisso com estes sistemáticos pagamentos busca reduzir os custos de oportunidade de investimentos que, de outra maneira, não seriam realizados privadamente devido aos seus reduzidos retornos líquidos esperados. No caso da economia brasileira, apesar do ceticismo quanto à eficácia das PPPs em promover suficiente volume de investimentos para reversão dos atuais gargalos na oferta de infra-estrutura, este mecanismo de parceria parece ter sido eleito, nos últimos anos, como uma das principais opções de escolha do governo central (e alguns governos estaduais) para elevar a precária oferta de infra-estrutura do Brasil.

Neste artigo analisamos os efeitos de longo prazo da lei de PPP brasileira. O modelo aqui proposto pressupõe a existência de dois tipos de capital. Um inerentemente privado, cujo retorno é plenamente apropriado privadamente, e um outro, denominado infra-estrutura, de propriedade do setor público ou privado, cujo retorno, devido suas características de bem público, apenas parcialmente pode ser apropriado pelo detentor de sua propriedade. Mais especificamente, seguindo a literartura da área (veja Turnovsky e Fisher (1995) e Glomm e Ravikumar (1997), entre muitos) admite-se a possibilidade da infra-estrutura gerar efeitos externos positivos sobre o conjunto da economia, seja sua propriedade pública ou privada. Adicionalmente, é suposto um governo que, além de investir, arrecada impostos, recebe renda de seus serviços e transfere renda aos indivíduos.

O modelo é calibrado para a economia brasileira buscando reproduzir os mecanismos da Lei das PPPs no Brasil. A calibragem dos parâmetros é feita de forma a haver uma correspondência entre a solução estacionária do modelo e os dados observados para a economia real. Neste sentido, a escolha dos indivíduos entre trabalho e lazer e entre consumo e poupança são determinantes para que as margens de receitas de impostos e suas distorções sejam bem capturadas. No presente modelo, especial atenção será dada à mensuração dos impactos da infra-estrutura sobre a economia.

Além disto, há que se definir qual a parcela dos serviços públicos de infra-estrutura é paga efetivamente pelo consumidor, o que em geral não é observado devido ao grande volume destes serviços que é ofertado gratuitamente e, portanto, não é contabilizado nas Contas Nacionais. Em nosso modelo só há um processo produtivo e, portanto, não existe separação entre firmas públicas e privadas. Estas são maximizadoras de lucro e cobram o valor de mercado pelo bens ofertados. Desta forma, há uma incompatibilidade entre o modelo e as Contas Nacionais. Nossa solução foi compensar as famílias pelo pagamento destes serviços com uma transferência de renda. Isto significou que esta parcela não paga pelos serviços de infra-estrutura pôde ser calibrada a partir da solução do modelo.

As simulações indicam que o impacto potencial da Lei das PPPs sobre o crescimento é pouco significativo. No longo prazo, no principal cenário simulado, o produto estaria $5 \%$ acima de sua tendência atual. Os ganhos de bem-estar estimados, entretanto, foram expressivos quando utilizada a calibração padrão. Entretanto, a magnitude destes ganhos varia consideravelmente quando se modifica na função de produção o efeito externo da infra-estrutura, um parâmetro de mensuração delicada e com pouco consenso na literatura.

O artigo está organizado da seguinte maneira. Na Seção 2 apresenta-se o modelo e em seguida, na Seção 3, descreve-se a calibragem de seus parâmetros. Na Seção 4 apresentam-se os principais resultados. E, finalmente, na Seção 5, destacam-se as principais conclusões. 


\section{O MODELO}

Supõe-se um bem final (correspondente ao agregado de bens e serviços oferecidos pelos setores privado e público em economias reais) produzido a partir da oferta total de trabalho e dos diferentes estoques de capital (privados ou público). Os estoques de capital são diferenciados e agrupados de acordo com suas capacidades em promover efeitos externos sobre o global da economia. Denomina-se estoque de capital de infra-estrutura, ou apenas "infra-estrutura", aquele estoque de capital capaz de gerar externalidades positivas. A oferta agregada restante de capital da economia é denominada apenas de "capital" e não promove nenhum efeito externo sobre a economia.

A função de produção agregada per capita desta economia é suposta ser expressa por:

$$
Y=A K^{\theta} G^{\phi} H^{1-\theta-\phi} \bar{G}^{\gamma}
$$

onde:

$A$ é um parâmetro de escala;

$\theta$, $\phi$ e $\gamma$ são parâmetros não negativos, tais que $\theta+\phi+\gamma<1$;

as variáveis agregadas per capita $Y, H, K$ e $G$ são, respectivamente, o produto ou renda total, o número de horas trabalhadas, o estoque de capital e o estoque de infra-estrutura e, por fim;

a expressão $\bar{G}^{\gamma}$ representa o efeito externo positivo (sobre a produtividade total dos fatores) promovido pelo estoque de infra-estrutura $G$, cuja intensidade é determinada pelo parâmetro $\gamma$. Esta especificação segue de perto Turnovsky e Fisher (1995) e Glomm e Ravikumar $(1994,1997)$ que assumem retornos constantes de escala aos fatores privados.

A equação (1) é a função de produção com que se depara a firma representativa, a qual, em cada instante $t$, escolhe os níveis de trabalho $(H)$, capital $(K)$ e infra-estrutura $(G)$ de forma a maximizar seu lucro, tomando como dados preços e a oferta de infra-estrutura per capita da economia $(\bar{G}){ }^{1}$

Portanto, o problema da firma representativa, para cada período $t$ é:

$$
\max _{H_{t}, K_{t}, G_{t}} A K_{t}^{\theta} G_{t}^{\phi} H_{t}^{1-\theta-\phi} \bar{G}_{t}^{\gamma}-w_{t} H_{t}-r_{t} K_{t}-r o_{t} G_{t}
$$

onde $w_{t}$ é o salário por hora trabalhada e $r_{t}$ e $r o_{t}$, respectivamente, são as taxas de aluguel do capital e da infra-estrutura.

Quanto à propriedade dos fatores de produção, supõe-se que $K$ seja pertencente ao setor privado, mas a oferta de infra-estrutura $(G)$ seja compartilhada entre famílias e o setor público. Note a suposição, simplificadora, de que todo o estoque de capital do setor público é, unicamente, infra-estrutura, o que, segundo a definição deste artigo, implica admitir que todo o estoque público de capital seja capaz de gerar efeitos externos positivos. Obviamente, isto pode não corresponder, exatamente, aos fatos em economias reais, mas parece ser uma suposição bastante razoável, na medida em que grande parte da oferta de capital público é composta de bens promotores de efeitos externos positivos, como ruas e auto-estradas, por exemplo.

Além disso, supõe-se que a propriedade da oferta de infra-estrutura (oferta pertencente ao setor privado ou público) não seja capaz de afetar a sua produtividade, ou seja, supõe-se que os estoques de infra-estrutura privada $(G p)$ e pública $(G g)$ sejam substitutos perfeitos. Neste caso a função de produção agregada poderia ser reescrita da seguinte forma:

$$
Y=A K^{\theta}(G p+G g)^{\phi} H^{1-\theta-\phi} \bar{G}^{\gamma}
$$

onde $\bar{G}=G p+G g$.

\footnotetext{
${ }^{1}$ Supõe-se um único bem produzido através de um único processo produtivo. Com efeito, a firma representativa descreve a média das atividades produtivas em curso na economia, incluindo, portanto, processos produtivos públicos e privados. Assim, a hipótese de maximização de lucros é, na verdade, uma hipótese simplificadora, na medida em que o setor público nem sempre persegue este objetivo.
} 
Supõe-se uma família ou consumidor representativo que vive infinitos períodos e, em cada período $t$, é dotado de uma unidade de tempo disponível para o trabalho $\left(h_{t}\right)$ e lazer $\left(1-h_{t}\right)$, extrai utilidade do consumo $\left(c_{t}\right)$, além do lazer, e desconta o futuro a um fator $\beta$ pertencente ao intervalo $(0,1)$ de acordo com a seguinte expressão: ${ }^{2}$

$$
U\left[c_{0}, c_{1}, \ldots, h_{0}, h_{1}, \ldots\right]=\sum_{t=0}^{\infty} \beta^{t}\left[\ln c_{t}+\psi \ln \left(1-h_{t}\right)\right]
$$

onde $\psi$ é uma constante expressando a importância relativa do lazer vis-à-vis o consumo.

Supõe-se, ainda, que a família representativa seja dotada no período $t$ de estoques acumulados de capital $\left(k_{t}\right)$ e infra-estrutura privada $\left(g p_{t}\right)$ e que suas rendas sejam compostas de renda do trabalho ofertado às firmas $\left(w_{t} h_{t}\right)$, renda obtida pelo aluguel às firmas dos estoques de capital $\left(r_{t} k_{t}\right)$ e infraestrutura privada $\left(r o_{t} g p_{t}\right)$ e renda auferida no recebimento de transferências do governo $\left(\Omega_{t}\right)$. Supõe-se, também, que todas estas fontes de renda, exceto transferências, sejam taxadas pelo governo e que a renda disponível em cada instante $t$ seja gasta em consumo $\left(c_{t}\right)$ e investimentos em bens de capital $\left(i_{t}\right)$ e infra-estrutura privada $\left(j p_{t}\right)$. Assim, a restrição orçamentária da família representativa em $t$ é:

$$
c_{t}+i_{t}+j p_{t} \leqslant(1-\tau h) w_{t} h_{t}+(1-\tau k) r_{t} k_{t}+(1-\tau g) r o_{t} g p_{t}+\Omega_{t}
$$

onde $\tau h, \tau k$ e $\tau g$, respectivamente, são as alíquotas de impostos sobre as rendas do trabalho, do capital e da infra-estrutura privada.

Admite-se que o consumidor conhece as leis de movimento, individuais e agregadas, dos estoques de capital e infra-estrutura:

$$
\begin{gathered}
k_{t+1}=(1-\delta) k_{t}+i_{t} \\
g p_{t+1}=(1-\delta g) g p_{t}+j p_{t} \\
K_{t+1}=(1-\delta) K_{t}+I_{t} \\
G_{t+1}=(1-\delta g) G_{t}+J p_{t}+J g_{t}
\end{gathered}
$$

onde $\delta$ e $\delta g$ são, respectivamente, as taxas de depreciação dos estoques de capital e infra-estrutura e $J p_{t}$ e $J g_{t}$, respectivamente, os investimentos agregados per capita privado e público em infra-estrutura.

Por fim, supõe-se que o consumidor toma todas as ações do governo como dadas e impõe-se que o governo esteja restrito a manter seu orçamento equilibrado para todo período $t$, ou seja, desconsiderase, aqui, a possibilidade de endividamento público. Assim, a restrição orçamentária per capita do governo em $t$ pode ser expressa por:

$$
\Omega_{t}+J g_{t}=T_{t}+r o_{t} G g_{t}, \forall t
$$

onde $\mathrm{ro}_{t} G g_{t}$ corresponde a receita proveniente do aluguel da infra-estrutura pública às firmas e $T_{t} \mathrm{o}$ total da receita corrente per capita de impostos determinada por:

$$
T_{t}=\tau h\left(w_{t} H_{t}\right)+\tau k\left(r_{t} K_{t}\right)+\tau g\left(r o_{t} G p_{t}\right) .
$$

\footnotetext{
${ }^{2}$ Neste artigo usam-se letras maiúsculas para variáveis agregadas, tomadas como dadas pelo consumidor representativo, e letras minúsculas para variáveis sobre as quais ele possui controle, a exceção são os preços que são supostos tomados como dados, apesar de estarem expressos em letras minúsculas.
} 
Tendo por objetivo um refinamento do processo de calibragem do modelo, torna-se relevante detalhar um pouco mais a equação (9). Note que, se todos os serviços públicos fossem pagos de acordo com seus custos de produção, a formulação acima seria compatível com as contas públicas reais. No entanto, isto, em geral, não é observado devido a substancial parcela de serviços públicos ofertados gratuitamente. Contudo, todos os serviços públicos, gratuitos ou não, como qualquer outro bem ou serviço, são produzidos a partir de trabalho, capital e infra-estrutura, organizados segundo algum processo produtivo específico (função de produção) e, portanto, existe um custo de oportunidade nesta produção que é pago ou pelos consumidores destes bens ou pelas firmas que os produzem (as firmas públicas no caso dos serviços públicos gratuitos).

Porém, neste artigo, por simplicidade, um único processo produtivo é suposto, e portanto, a separação entre firmas públicas e privadas não é possível. Diante disto, optou-se pela suposição de que todas as firmas comportem-se como firmas maximizadoras de lucro e cobrem o valor de mercado pela sua oferta de produção. Por esta razão, na restrição orçamentária do governo, aparece a renda do aluguel da infra-estrutura pública às firmas $(r o G g)$ e, no problema do consumidor, todos os bens e serviços privados ou públicos são pagos. Entretanto, como em economias do mundo real, grande parte da oferta de serviços públicos é gratuita ou subsidiada (como os serviços de transporte, por exemplo), a solução encontrada foi compensar as famílias pelo pagamento destes serviços através de uma transferência de renda correspondente a estes gastos. Com efeito, o termo $\Omega_{t}$ nas equações (9) e (4) incorpora o total das despesas do governo com a oferta gratuita destes bens e serviços públicos, além das transferências, propriamente ditas, de recursos financeiros às famílias, ou seja:

$$
\Omega_{t}=T R_{t}+\alpha\left(r o_{t} G g_{t}\right)
$$

onde a expressão $\alpha\left(r o_{t} G g_{t}\right)$, com $\alpha \in[0,1]$, corresponde ao custo de oportunidade incorrido pelo governo por este abrir mão de parte, mas não completamente, da receita do aluguel da infra-estrutura pública às firmas em favor das famílias. $T R_{t}$ seria o restante das transferências públicas per capita às famílias ou o que, em economias reais, poderia se chamar gastos correntes per capita do setor público.

Uma formulação mais próxima das efetivas despesas e receitas do governo em economias reais seria alcançada com:

$$
T R_{t}+J g_{t}=T_{t}+(1-\alpha) r o_{t} G g_{t}, \forall t,
$$

onde $(1-\alpha)$ interpreta-se como a parcela do custo de capital da infra-estrutura pública que é efetivamente paga pelas famílias. O lado direito da equação (11) perfaz o que se conhece como total da receita líquida do governo ou setor público. ${ }^{3}$

A partir da restrição orçamentária do governo (11), um passo final para caracterizar uma política fiscal seria especificar uma regra para distribuição das receitas do governo entre transferências e investimentos públicos, a qual supõe-se dada por:

$$
\begin{gathered}
T R_{t}=\alpha_{0} T_{t}+\left(1-\alpha_{1}\right)(1-\alpha) r o_{t} G g_{t} \\
J g_{t}=\left(1-\alpha_{0}\right) T_{t}+\alpha_{1}(1-\alpha) r o_{t} G g_{t}
\end{gathered}
$$

onde $\alpha_{0}$ é fração da receita corrente de impostos dedicada aos gastos correntes do setor público e $\alpha_{1}$ a fração da receita auferida pelo efetivo aluguel da infra-estrutura pública dedicada ao financiamento do investimento público.

\footnotetext{
${ }^{3}$ Admitindo-se a hipótese simplificadora de que todos os serviços ofertados pela administração pública são gratuitos, enquanto os serviços prestados pelas empresas públicas são pagos de acordo com seu valor de mercado, pode-se concluir que dado o significado acima para o parâmetro $\alpha$, a parcela $(1-\alpha)$ ro $G g$ do total da renda do setor público pode ser interpretada como receita gerada pelo excedente das empresas públicas.
} 
Define-se o vetor $\tau=\left\{\alpha, \alpha_{0}, \alpha_{1}, \tau h, \tau k, \tau g\right\}$ como uma política fiscal do governo.

Assim, o problema do consumidor na sua forma recursiva pode ser descrito através das seguintes equações de otimalidade: ${ }^{4}$

$$
v(K, k, G, g p)=\max _{c, h, i, j p}\left[\ln c+\psi \ln (1-h)+\beta v\left(K^{\prime}, k^{\prime}, G^{\prime}, g p^{\prime}\right)\right]
$$

sujeito a

$$
\begin{aligned}
& c+i+j p=(1-\tau h) w(K, G, \tau) h+(1-\tau k) r(K, G, \tau) k \\
& +(1-\tau g) \operatorname{ro}(K, G, \tau) g p+\Omega(K, G, \tau) \\
& k^{\prime}=(1-\delta) k+i \\
& g p^{\prime}=(1-\delta g) g p+j p \\
& K^{\prime}=(1-\delta) K+I(K, G, \tau) \\
& G^{\prime}=(1-\delta g) G+J p(K, G, \tau)+J g(K, G, \tau)
\end{aligned}
$$

dados os preços, $k_{0}$ e $g p_{0}>0, c \geq 0$ e $0 \leq h \leq 1$.

Dados os estados individuais $s=(K, k, G, g p)$ e estados agregados $S=(K, G)$, onde $G=G p+$ $G g$, um Equilíbrio Competitivo Recursivo para esta economia é um conjunto de regras individuais de decisão, $c(s), i(s), j p(s), h(s)$, um conjunto de regras agregadas de decisão per capita $C(S), I(S)$, $J p(S), H(S)$, funções para os preços dos fatores $w(S), r(S)$, ro $(S)$ e uma função valor $v(s)$ tais que satisfazem: a) o problema do consumidor (14); b) o problema das firmas (2); c) a consistência entre a decisões individuais e agregadas per capita, i.e., $C(S)=c(s), I(S)=i(s), J p(S)=j p(s)$ e $H(S)=h(s)$ quando $k=K, g p=G p$; d) o orçamento equilibrado do governo; e e) a restrição de recursos da economia, $C(S)+I(S)+J p(S)+J g(S)=Y(S)=A K^{\theta} G^{\phi} H(S)^{1-\theta-\phi} \bar{G}^{\gamma}, \forall S$.

\section{CALIBRAÇÃO}

A calibragem dos parâmetros é feita de forma a haver uma correspondência entre a solução estacionária do modelo e os dados observados para a economia real, supondo que esta economia esteja em trajetória estacionária. A solução estacionária do modelo caracteriza-se por uma política fiscal estacionária para todo $t$, acompanhada de estoques de capital e infra-estrutura per capita, tal como níveis de consumo, horas trabalhadas e produto per capita, igualmente, invariantes ao longo do tempo. Neste sentido, o primeiro passo do processo de calibragem seria fazer um mapeamento entre os dados da contabilidade nacional do Brasil e as variáveis do modelo; e o segundo passo, a partir desta contabilidade do modelo, determinar o seu conjunto de parâmetros, o qual divide-se em: i) parâmetros de preferência ( $\beta$ e $\psi$ ); ii) parâmetros de tecnologia $(\delta, \delta g, \theta, \phi, A$ e $\gamma)$; e iii) parâmetros da política estacionária básica ou atual do governo $\left(\alpha, \alpha_{0}, \alpha_{1}, \tau g, \tau k\right.$ e $\left.\tau h\right) .^{5}$

\footnotetext{
${ }^{4}$ Usa-se $x^{\prime}$ para indicar a variável no próximo período.

${ }^{5} \mathrm{~A}$ solução estacionária para as variáveis per capita, como função dos parâmetros do modelo, é obtida aplicando-se sobre o conjunto de condições de primeira ordem do problema do consumidor e firmas, leis de movimento dos capitais, além da restrição orçamentária do governo, a condição de uma política fiscal estacionária e variáveis per capita inalteradas ao longo do tempo.
} 
O mapeamento entre variáveis artificiais e dados, em geral, não é imediato. Devido à forma como determinados agregados são conceituados e medidos na contabilidade nacional (CN), necessita-se um procedimento de ajuste entre as informações disponíveis e as variáveis do modelo.

A primeira grande diferença entre a $\mathrm{CN}$ e o modelo é que, na primeira, o consumo de bens duráveis é contabilizado como gastos de consumo das famílias, enquanto no modelo, dado o caráter de capital fixo destes bens, estes são considerados como parte do investimento e somente os serviços gerados por estes bens de capital são considerados consumo. Implicitamente, na função de produção agregada do modelo, existe um setor que oferta serviços domésticos às famílias. No modelo, as famílias investem em bens de consumo duráveis com a finalidade de alugá-los às firmas, as quais vendem seus serviços de volta às famílias. Este problema, além de exigir uma realocação entre os gastos de consumo e investimento, provoca uma subestimação no cálculo do produto da economia, na medida em que existem serviços domésticos que não estão sendo devidamente contabilizados. Portanto, o valor do produto total da economia deve sofrer um ajuste para cima.

Um ajuste semelhante deve ser feito considerando-se a forma como a oferta de serviços públicos é contabilizada. No modelo, devido à hipótese simplificadora de um único setor produtivo, todos os serviços privados ou públicos são produzidos pela firma representativa e, portanto, vendidos pelo valor de mercado. $\mathrm{Na} \mathrm{CN}$, entretanto, a mensuração da oferta dos serviços públicos, particularmente aqueles serviços para os quais não existe um valor de mercado, é feita, em geral, a partir de seus custos de produção, entre os quais está a depreciação do capital envolvido neste processo de produção. Contudo, haveria também a necessidade de imputar, nestes custos de produção, o custo de oportunidade do retorno líquido deste capital, apesar deste custo não ser contabilizado. Portanto, novamente, observase uma subestimação no cálculo do produto da economia, o qual, para que se torne compatível com o valor relativo ao modelo deve sofrer um ajuste para cima. Levando-se em conta estes dois aspectos pode-se verificar que o nível de produto estimado, no caso da economia americana, em média para a década de noventa, eleva-se em torno de $10 \%$ acima daquele medido pela contabilidade nacional dos EUA (NIPA).

Diante destas observações, apesar da inexistência de dados apropriados para se verificar a correta dimensão destas distorções no caso da economia brasileira, considerou-se que, diante da possibilidade destas subestimações não serem desprezíveis, talvez fosse adequado encontrar alguma forma de ajuste. No processo de calibragem, os valores atribuídos às relações consumo-produto e investimento-produto público e privado correspondem aos seus respectivos valores observados na $\mathrm{CN}$ do Brasil, porém, ajustados por fatores de correção obtidos no efetivo ajuste destas variáveis para a economia americana, utilizando-se informações da NIPA. ${ }^{6}$ Por exemplo, segundo dados do Instituto Brasileiro de Geografia e Estatística (IBGE), a média do agregado do consumo das famílias e governo, medido em relação ao PIB, entre 1999 e 2003, corresponde a 0,792. Ajustando-se este valor, para a calibragem do modelo, de acordo com o procedimento acima descrito, obtém -se: $C / Y=0,733$. A redução procura eliminar, em parte, a superestimação do valor deste agregado na $\mathrm{CN}$, devido ao fato do consumo de bens duráveis estar a ele sendo adicionado.

Devido limitações de informações para a economia brasileira, decidiu-se o seguinte procedimento geral para calibragem do modelo. Para os parâmetros de tecnologia $(\delta, \delta g, \theta$ e $\phi)$ e preferência $(\beta$ e $\psi)$ utilizar informações anuais da NIPA, supondo que tais parâmetros sejam os mesmos entre as economias brasileira e americana. Entretanto, para o conjunto de parâmetros de política do governo, utilizar, além dos demais parâmetros, somente informações anuais relativas à $\mathrm{CN}$ do Brasil.

Na solução estacionária do modelo $\delta g=J g / G g$, como, segundo dados anuais da NIPA, o investimento bruto e o estoque de capital do governo, como proporção do produto, em média para os anos noventa, correspondem, respectivamente, a 0,033 e 0,604 , admite-se $\delta g=0.054$.

\footnotetext{
${ }^{6}$ Um completo detalhamento dos ajustes entre as informações da NIPA e as variáveis do modelo apresentado na seção anterior está presente em Pereira (2006).
} 
Da mesma forma, na solução estacionária do modelo $\delta=I / K$, onde tanto o investimento $(I)$ como o estoque de capital $(K)$ não incluem bens de capital em infra-estrutura. Para o cálculo de $\delta$, portanto, deve-se fazer a dicotomia dos agregados do investimento bruto privado $(I+J p)$ e estoque de capital privado $(K+G p)$ da economia. O valor agregado do estoque total de capital privado, como proporção do produto, $(K+G p) / Y$, segundo dados anuais da NIPA, em média para os anos noventa, é 2,452 . Portanto, obtém-se $K / Y=2,199$, arbitrando-se o valor agregado do estoque de capital de infra-estrutura, como proporção do produto, $G p / Y$, em média para os anos noventa, igual a $0,253 .{ }^{7}$

De acordo com o modelo, o produto total da economia destina-se, unicamente, ao consumo $(C)$, investimento público $(J g)$ e investimentos privados $(I+J p)$. O investimento bruto do governo corresponde àquele informado na NIPA. O consumo é contabilizado incluindo gastos do governo, excluindo consumo de bens duráveis e imputando-se a ele os devidos serviços, anteriormente mencionados. Por consequência, a soma dos investimentos privados incluem, além do consumo de bens duráveis, as exportações líquidas de bens e serviços. Medido desta maneira, o valor total do investimento privado, em proporção ao produto, $(I+J p) / Y$, em média para os anos noventa, segundo a NIPA é 0,223 . Admitindo-se que na solução estacionária do modelo $J p / Y=\delta g(G p / Y)$, obtém-se $I / Y=0,210$ e, portanto, $\delta=0,095$.

Como mencionado acima, uma vez imputados os serviços relativos aos bens de consumo duráveis e capital do governo, o produto da economia americana, em média para os anos noventa, eleva-se em torno de $10 \%$. Levando-se em conta estes ajustes nos dados da NIPA, neste período, a participação média da renda do capital equivale a 0,433 . Portanto, segundo o modelo, este valor deve corresponder à soma dos parâmetros $\theta$ e $\phi$. A separação entre as rendas do capital $(K)$ e infra-estrutura $(G)$ é obtida dada a relação $G / Y=0,773$ (soma de $G p / Y$ e $G g / Y$, ajustada para o novo nível de produto), supondo a taxa de juros líquida anual (i) para a economia americana em torno de $4 \%$ e admitindo-se $r o=i+\delta g$. Como, de acordo com o modelo, $\phi=r o G / Y$, obtém-se $\phi=0,074$ e, consequentemente, $\theta=0,359$.

O valor para o parâmetro $\beta=0,961$ é obtido a partir da hipótese de uma taxa de juros líquida anual para a economia americana em torno de $4 \%$. O parâmetro $\psi=1,056$ obtém-se, na calibragem do modelo para a economia americana, admitindo-se que as famílias alocam ao trabalho cerca de um terço de suas horas disponíveis.

Partindo-se para a calibragem dos parâmetros de política do modelo, deve-se lembrar que $\alpha$ representa a fração gratuita do total dos serviços públicos ofertados. O total de serviços públicos divide-se entre serviços ofertados pelas empresas públicas e administração pública e, por simplicidade, supõe-se que os serviços públicos gratuitos correspondam àqueles ofertados pela administração pública. Assim, admitindo-se a divisão da oferta total de capital público $(G g)$ em capital das empresas públicas $(G E g)$ e administração pública $(G G g)$ e considerando-se a proporcionalidade entre a oferta de serviços públicos e o seu respectivo estoque de capital, de acordo com o modelo, obtém-se: $\alpha /(1-\alpha)=G G g / G E g$. Uma vez que se admitam taxas de depreciação idênticas para ambos os estoques de capital, determina-se: $\alpha /(1-\alpha)=J G g / J E g$ ou $\alpha=J G g /(J G g+J E g)$, onde $J G g$ são os investimentos da administração pública e $J E g$ os investimentos das empresas públicas. Tomando $J G g /(J G g+J E g)$ como a média do período 1999-2003 para a participação da formação bruta de capital da administração pública no investimento total do setor público no Brasil, calculada a partir de dados divulgados pelo Instituto de Pesquisa Econômica Aplicada (IPEA), obtém-se $\alpha=0,602$.

Através das condições de primeira ordem do problema do consumidor e firmas e leis de movimento dos capitais, em estado estacionário, obtêm-se expressões para os parâmetros de política $(\tau g, \tau k$ e $\tau h)$ em função dos parâmetros de tecnologia $(\delta, \delta g, \theta$ e $\phi)$ e preferência $(\beta$ e $\psi)$ e valores estacionários para

\footnotetext{
${ }^{7} \mathrm{O}$ valor para $G p / Y=0,253$ foi extraído da contabilidade da BEA (Bureau of Economic Analysis, Table 2.1. Current-Cost Net Stock of Private Fixed Assets, Equipment and Software, and Structures by Type, http://www.bea.gov/bea/dn/FA2004/SelectTable.asp) e corresponde à média para os anos noventa dos valores, em relação ao produto, dos seguintes tipos de capital: 1) equipment and software: computers and peripheral equipment, software, communication equipment, transportation equipment; 2) structures: communication, educational, railroads and other structures (consisting primarily of streets, dams and reservoirs, sewer and water facilities, parks, and airfields).
} 
as relações consumo-produto $(C / Y)$ e investimento-produto $(J g / Y, J p / Y$ e $I / Y)$, além do número de horas trabalhadas $(H)$.

Considerando-se a necessidade de realocação do consumo de bens duráveis para investimento e a incorporação no consumo e produto dos serviços relativos a estes bens, tal como de parte do capital do governo, obtêm-se, após os devidos ajustes: i) $C / Y=0,733$ a partir da média entre 1999 e 2003 da soma do consumo das famílias e do governo, medidos em relação ao produto, correspondente a 0,792 , segundo informações do Instituto Brasileiro de Geografia e Estatística (IBGE); ii) $\mathrm{Jg} / Y=0,029$ através da média entre 1999 e 2003 do total do investimento público, medido em relação ao produto, igual a 0,032, de acordo com dados divulgados pelo Instituto de Pesquisa Econômica Aplicada; iii) $\mathrm{Jp} / Y=$ 0,010, admitindo-se como investimento privado total em infra-estrutura os investimentos privados médios do período 1990-1998, medidos em relação ao produto, em estradas, ferrovias, setores elétrico, de água e telecomunicações, divulgados pelo Banco Mundial, equivalentes a $0,0107^{8}$; iv) $H=0,3$, seguindo Cooley e Prescott (1995); e v) $I / Y=0,228$ dado que, de acordo com o modelo, em equilíbrio tem-se $Y=C+I+J p+J g$.

Impondo-se as condições de estado estacionário sobre as equações de movimento dos estoques de capital e condições de primeira ordem dos problemas da firma e consumidor, obtém-se o conjunto de taxas da política pública em função dos demais parâmetros (de preferência e tecnologia), das horas de trabalho $(H)$, relação consumo-produto $(C / Y)$ e relações investimento-produto $(I / Y, J p / Y$ e $J g / Y)$. Os valores encontrados para $\tau g$, $\tau k$ e $\tau h$ foram, respectivamente, $0,073,0,091$ e 0,416 .

Por simplicidade, admite-se $\alpha 1=1-\alpha 0$ (vide equações 12 e 13), o que significa que as duas fontes de receita do governo distribuem-se na mesma proporção entre investimentos e gastos correntes. Aplicando-se sobre a equação (13), que descreve os investimentos públicos, as condições de primeira ordem do problema das firmas e supondo a economia em trajetória estacionária, obtém $\alpha 0$ como função dos parâmetros de tecnologia $(\theta$ e $\phi)$, parâmetros de política $(\alpha, \tau g, \tau k$ e $\tau h)$ e relações investimentoproduto $(J g / Y$ e $J p / Y)$. Dado os valores acima para estes parâmetros e relações, encontra-se $\alpha_{0}=$ 0,900 .

Obtidos os valores para os parâmetros de política pública, restam, ainda, dois últimos parâmetros a serem calibrados, ambos relacionados à função de produção (1). O primeiro deles, o parâmetro de escala $A$ tem como única finalidade ajustar o valor do produto de forma a torná-lo unitário.

O segundo parâmetro, $\gamma$, não pode ser obtido diretamente através da contabilidade nacional. Em geral, seu valor é estimado econometricamente, mas não há consenso aqui, embora se aceite na maioria dos casos valores positivos e significativos para este. As estimativas conjuntas de $\gamma$ e $\phi$ variam, em sua maioria, entre zero, (Holtz-Eakin, 1992), algo em torno de 0,10 (Duffy-Deno e Eberts, 1991, Baffes e Shah, 1998) ou 0,16 (Calderón e Servén, 2003, Ai e Cassou, 1995), embora alguns poucos trabalhos tenham estimado valores superiores. A média das estimações em Ferreira e Araújo (2007), que trabalham com dados brasileiros, é de 0,12 . Dado o valor de $\phi(=0,074)$, decidiu-se utilizar um valor intermediário para $\gamma$ como valor básico, $\gamma=0,05$, embora também sejam reportadas simulações para $\gamma=0$ e $\gamma=0,10$. O resumo de todos os valores dos parâmetros está apresentado na Tabela 1 abaixo.

Tabela 1 - Parâmetros calibrados

\begin{tabular}{|l|c|c|c|c|c|c|}
\hline \multicolumn{2}{|c|}{ Preferência } & \multicolumn{5}{|c|}{ Tecnologia } \\
\hline$\beta$ & $\psi$ & $\delta$ & $\delta g$ & $\theta$ & $\phi$ & $\gamma$ \\
0,961 & 1,056 & 0,095 & 0,054 & 0,359 & 0,074 & 0,05 \\
\hline \multicolumn{7}{|c|}{ Política Básica ou Atual do Governo } \\
\hline$\alpha$ & $\alpha 0$ & $\alpha 1$ & $\tau g$ & $\tau k$ & $\tau h$ & \\
0,602 & 0,900 & 0,100 & 0,073 & 0,091 & 0,416 & \\
\hline
\end{tabular}

\footnotetext{
${ }^{8} \mathrm{O}$ dado mencionado foi obtido no site do Banco Mundial: http://wbln1018.worldbank.org/LAC/LAC.nsf/
} ECADocByUnid/9A886DFD517053AB85256D440002B206?Opendocument 


\section{RESULTADOS}

O principal objetivo desta seção é verificar os efeitos alocativos e de bem estar social desencadeados por eventuais mudanças na política básica do governo, definida por $\left(\alpha, \alpha_{0}, \alpha_{1}, \tau g, \tau k\right.$ e $\left.\tau h\right)$, onde os valores dos parâmetros correspondem aos do equilíbrio estacionário do modelo expressos na Tabela 1. Mais precisamente, determinar como mudanças particulares na política básica afetariam os valores de longo prazo do modelo e como as trajetórias de transição das principais variáveis do modelo modificariam os níveis de bem estar das famílias.

A medida de bem estar equivale ao percentual constante de mudança no consumo, $\mathbf{x}$, relativo aos níveis correspondentes à política básica (BP) para todos os momentos do tempo, $t$, mantidas as horas de trabalho em seus níveis de política básica, requerido para manter o nível de utilidade igual àquele obtido no experimento da política alternativa (AP). Assim, a medida de bem estar $x$ deve satisfazer a seguinte equação:

$$
\sum_{t=0}^{\infty} \beta^{t}\left[\ln \left(C_{t}^{B P}(1+x)\right)+A \ln \left(1-H_{t}^{B P}\right)\right]=\sum_{t=0}^{\infty} \beta^{t}\left[\ln C_{t}^{A P}+A \ln \left(1-H_{t}^{A P}\right)\right]
$$

onde, $H_{t}^{B P}$ e $H_{t}^{A P}$ são as horas de trabalho e $C_{t}^{B P}$ e $C_{t}^{A P}$ são os níveis de consumo escolhidos em cada instante do tempo $t$, supondo-se, respectivamente, a política básica e a política alternativa. Esta medida é tradicional na literatura e segue, entre muitos, Lucas (1987) e Cooley e Hansen (1992).

\subsection{Política de Parceria Público-Privada}

A Lei 11.079 de 31 de dezembro de 2004 instituiu normas gerais para licitação e contratação da chamada parceria público-privada no Brasil. Uma Parceria Público-Privada (PPP) pode ser definida como um contrato de concessão envolvendo pagamentos do setor público ao parceiro privado em contrapartida à oferta privada de serviços públicos.

A motivação para tal parceria deve-se ao reconhecimento de que o retorno privado líquido do investimento em bens públicos, em geral, é inferior ao seu retorno social líquido, sendo, portanto, justificável compensar o setor privado pelo investimento em tais ativos. De forma resumida, o objetivo principal da lei é fazer uso das PPPs como mecanismo de atração do setor privado para investimentos em infra-estrutura (definindo-se infra-estrutura como o estoque de capital capaz de gerar externalidades positivas). Porém, segundo a Lei das PPPs, o volume de recursos públicos, destinados a elevar o retorno líquido do capital privado investido, limita-se a somente $1 \%$ da receita corrente líquida do exercício. 0 que acaba por suscitar dúvidas sobre sua efetiva capacidade de atrair suficiente investimento privado para reverter o consensual gargalo existente na atual oferta de infra-estrutura no Brasil.

Uma Política PPP, anunciada no período $t=0$, é definida pelo aumento do retorno líquido privado do capital de infra-estrutura através da imposição de valores negativos para a taxa $\tau g$, sujeito à restrição de que este dispêndio adicional do governo seja exatamente igual a $1 \%$ de sua receita corrente líquida a todo momento.

Na Tabela 2, admitindo-se $\gamma=0,05$, encontram-se os impactos de crescimento de longo prazo determinados pela Política PPP, além de seu efeito sobre o nível de bem estar social medido através de $x \%$.

Os resultados indicam que o efeito positivo da política, na calibração padrão, embora não espetacular, é relevante. No longo prazo o programa de PPP, quando operando a plena capacidade, possui potencial para aumentar em $5 \%$ o produto em relação a sua tendência atual. Isto é, tudo mais constante o PIB ficará cinco por cento acima do que ficaria sem esta política. Ao mesmo tempo, o consumo ficaria, aproximadamente, $4 \%$ acima da tendência observada na política básica atual e, vale destacar, haveria um estrondoso efeito sobre o crescimento dos investimentos privados em infra-estrutura. Este praticamente dobraria de valor, sugerindo o forte potencial de eficácia da Política de PPP em expandi-los. 
Tabela 2 - Política de parceria público-privada (variação \%)

\begin{tabular}{|c|c|}
\hline$Y$ & 5,0 \\
\hline$C$ & 3,9 \\
\hline$I$ & 5,0 \\
\hline$J_{P}$ & 92,4 \\
\hline$J_{g}$ & 2,0 \\
\hline$T f$ & 2,0 \\
\hline Bem-Estar $(x \%)$ & 1,76 \\
\hline
\end{tabular}

Obviamente estamos ignorando incertezas diversas neste processo que podem deprimir a participação privada e assumindo que todos os projetos serão igualmente produtivos. Assim, este resultado, provavelmente, corresponde ao limite superior dos possíveis benefícios proporcionados pela PPP, mas de qualquer forma, no cenário acima, o impacto é relevante.

Note, também, que o ganho de bem-estar apresentado na última linha é significativo. Em valor presente, o aumento do consumo ao longo do tempo seria algo em torno de 1,8\% ao ano. Em termos comparativos, Lucas (1987), por exemplo, estima que os ganhos de suavização do ciclo nos Estados Unidos ficam em torno de $0,1 \%$ do consumo total, ou $0,3 \%$ quando se considera choques indiossincráticos e restrição à liquidez (Imrohoruglu, 1989). ${ }^{9}$ No Brasil, Araújo e Ferreira (1999) estimam que uma reforma tributária que reduzisse taxação sobre investimento e renda dos fatores, aumentando compensatoriamente impostos sobre o consumo geraria um ganho de bem-estar de $0,6 \%$ (1,1\% com uma proposta mais agressiva). Em termos comparativos, portanto, o ganho estimado da política de PPP é expressivo.

Deve-se destacar que os resultados, obviamente, dependem fortemente do parâmetro $\gamma$. Uma política de parceria público-privada é tanto mais relevante para o bem estar social, quanto maiores forem as externalidades positivas do estoque de infra-estrutura. Simulando-se para o caso onde não há qualquer externalidade, $\gamma=0$, o efeito de bem estar da política é bem inferior (somente $0,39 \%$ ) e este valor positivo encontrado é explicado, unicamente, pela redução do parâmetro $\tau g$ que distorcia negativamente as decisões de investimentos em infra-estrutura. Mais ainda, neste caso o nível do produto no longo prazo seria somente $3 \%$ superior ao seu valor observado segundo a tendência atual. Por outro lado, com $\gamma=0,1$, o produto no "steady state" seria $7,5 \%$ superior e o ganho de bem-estar corresponderia a $3,3 \%$. Note, entretanto, que na calibração padrão $(\gamma=0,05)$ já estamos assumindo que a soma do efeito externo e interno da infra-estrutura é $\gamma+\phi=0.124$, em linha com estimações para a economia brasileira encontradas em Ferreira e Issler (1998) e Ferreira e Araújo (2007). Portanto, $\gamma=0,1$ estaria um pouco acima dos valores aceitáveis.

A partir das simulações, conclui-se da Política de PPP seu robusto potencial de expansão da participação privada na oferta de infra-estrutura, e seu impacto de crescimento sobre a soma dos investimentos privados e públicos em infra-estrutura, medidos em relação ao produto, da ordem de $19 \%$. Simulando uma aprovação de um novo limite imposto por Lei para o comprometimento com gastos públicos em PPP de $1 \%$ para $2 \%$, o impacto desta nova Política de PPP sobre a expansão dos investimentos públicos e privados em infra-estrutura, em relação ao produto, seria bem mais expressiva, alcançando $27 \%$ na calibração padrão $(\gamma=0,05)$. Os impactos de longo prazo sobre o crescimento do produto e consumo, em relação aos seus valores atuais, ficariam em torno de $7 \%$ e $5,5 \%$, respectivamente, e o ganho de bem-estar, aproximadamente, $2,4 \%$, sem dúvida, um ganho bastante significativo. No caso $\gamma=0$, entretanto, o ganho de bem-estar corresponderia somente a $0,5 \%$.

\footnotetext{
${ }^{9}$ No último caso, pode chegar a 1,5\% para parâmetros de aversão ao risco mais altos, mas volta a ser insignificante quando empréstimos, mesmo que a um custo alto, são reintroduzidos.
} 


\section{CONCLUSÃO}

Os resultados das simulações realizadas indicam que os efeitos positivos sobre o bem estar, ou a eficiência econômica, de uma Política de Parceria Público-Privada (PPP) dependem fortemente do grau de intensidade exercido pelo estoque de infra-estrutura sobre a produtividade total dos fatores de produção (parâmetro $\gamma$ ). Parâmetro cujo valor, infelizmente, não é consensual, apesar de evidências indicando sua positividade.

Os resultados sugerem que uma Política de PPP é tanto mais relevante para o bem estar social, quanto maiores forem as externalidades positivas promovidas pelo estoque total de infra-estrutura ou, o que seria equivalente, quanto maiores forem as diferenças entre os retornos sociais e privados deste estoque. Na calibração que julgamos mais adequada e metodologicamente defensável, supondo externalidades positivas $(\gamma=0,05)$, a Política de PPP implica crescimento pouco expressivo, entretanto, seu efeito de bem estar é significativo, comparado a efeitos de políticas obtidos por outros autores.

A calibração do modelo para o Brasil procurou mensurar com cuidado certas particularidades da restrição orçamentária do governo, até onde se sabe, inexploradas por outros autores, como a mensuração da parcela dos serviços públicos de infra-estrutura que não é efetivamente paga pelas famílias. Consideramos, portanto, o esforço de calibração do modelo uma contribuição relevante deste artigo.

\section{Referências Bibliográficas}

Ai, C. \& Cassou, S. (1995). A normative analysis of public capital. Applied Economics, 27:1201-1209.

Araújo, C. H. V. \& Ferreira, P. C. (1999). Reformas tributárias no Brasil: Efeitos alocativos e impactos de bem-estar. Revista Brasileira de Economia, 53(2):133-166.

Baffes, J. \& Shah, A. (1998). Productivity of public spending, sectorial allocation choices, and economic growth. Economic Development and Cultural Change, 46:291-303.

Calderón, C. \& Servén, L. (2003). The output cost of latin america's infrastructure gap. In Easterly, W. \& Servén, L., editors, The Limits of Stabilization: Infrastructure, Public Deficits, and Growth in Latin America. Stanford University Press and World Bank.

Cooley, T. F. \& Hansen, G. (1992). Tax distortion in a neoclassical monetary economy. Journal of Economic Theory, 58:290-316.

Cooley, T. F. \& Prescott, E. (1995). Economic growth and business cycles. In Cooley, T., editor, Frontiers of Business Cycles Research. Princeton Press.

Duffy-Deno, K. \& Eberts, R. W. (1991). Public infrastructure and regional economic development: A simultaneous equations approach. Journal of Urban Economics, 30:329-43.

Ferreira, P. C. \& Araújo, C. H. V. (2007). Growth and fiscal effects of infrastructure investment in Brazil. In Guillermo Perry, L. S. \& (Org.), R. S., editors, Fiscal Policy, Stabilization, and Growth. The World Bank, Washington.

Ferreira, P. C. \& Issler, J. V. (1998). Time series properties and empirical evidence of growth and infrastructure. Revista de Econometria, 18:31-71.

Glomm, G. \& Ravikumar, B. (1994). Public investment in infrastructure in a simple growth model. Journal of Economic Dynamics and Control, 18:1173-1187.

Glomm, G. \& Ravikumar, B. (1997). Productive government expenditures and long-run growth. Journal of Economic Dynamics and Control, 21:183-204. 
Holtz-Eakin, D. (1992). Public sector capital and productivity puzzle. NBER Working Paper no. 4122.

Imrohoruglu, A. (1989). Cost of business cycles with indivisibilities and liquidity constraint. Journal of Political Economy, 97:1364-1383.

Lucas, R. (1987). Models of Business Cycles. Basil Blackewell, Cambridge, MA.

Pereira, R. A. C. (2006). Políticas públicas para a infra-estrutura. Tese de Doutorado - Escola de Pós Graduação em Economia, Fundação Getúlio Vargas, Rio de Janeiro.

Turnovsky, S. J. \& Fisher, W. H. (1995). The composition of government expenditure and its consequences for macroeconomic performance. Journal of Economic Dynamics and Control, 19(4):747-786. 
